\title{
Propuesta para la consolidación de una Red Chilena de Estudios Socio-Ecológicos de Largo Plazo
}

\author{
Strategic plan to consolidate the Chilean Long-Term Socio-ecological Research Network
}

\author{
Mariela Núñez-Ávila a,b,*, Cristian Frêne b,c, Juan J Armesto ${ }^{\text {b,c }}$ \\ *Autor de correspondencia: aUniversidad de Concepción, Facultad de Ciencias Forestales, \\ Departamento Manejo de Bosques y Medio Ambiente, Victoria 631, Concepción, Chile, tel.56-412661437, mnuneza@udec.cl \\ ${ }^{b}$ Instituto de Ecología y Biodiversidad, Las Palmeras 3425, Ñuñoa, Santiago, Chile. \\ ${ }^{c}$ Pontificia Universidad Católica de Chile, Departamento de Ecología, Alameda 340, Santiago, Chile.
}

\begin{abstract}
SUMMARY
Humans are causing profound and rapid transformations of Earth's ecosystems to the extent that we are threatening the sustainability of the biosphere. The development of long-term socio-ecological research, integrating monitoring and experiments at the ecosystem scale, will be essential to detect, prevent, and mitigate the degradation of ecosystems at regional and global scales. To strengthen and expand the nascent Long-Term Socio-ecological Research (LTSER) Network of Chile, we need to integrate efforts of academic, private, and government institutions. A strong long-term research and monitoring program will require a conceptual framework and a set of relevant, comparative, and trans-disciplinary questions. Here, we identify these relevant questions and propose a conceptual framework for the Chilean LTSER Network. Further, we provide guidelines for its organization and consider the needs for infrastructure and funding to sustain the operation of a national network of ecological observatories.
\end{abstract}

Key words: socio-ecological studies, global change, human wellbeing, policy making.

\section{RESUMEN}

El ser humano ha desencadenado profundas y rápidas transformaciones de los ecosistemas afectando su capacidad de mantener los procesos y funciones que sustentan la biosfera. El desarrollo de investigación socio-ecológica de largo plazo, incluyendo monitoreo y experimentación, es esencial para detectar, prevenir y mitigar la degradación de los ecosistemas a escala local, regional y global. El fortalecimiento y expansión de una Red de sitios de Estudios Socio-Ecológicos de Largo Plazo (LTSER) en Chile requiere integrar los esfuerzos de una diversidad de instituciones académicas, públicas y privadas. La investigación socio-ecológica en esta red de sitios requiere además un marco conceptual de preguntas relevantes y comparativas que sean abordadas desde diversas disciplinas. En este trabajo se identificaron las preguntas relevantes para el desarrollo de la Red LTSER en Chile, se presenta un marco conceptual, una propuesta de organización, y se discuten las necesidades de infraestructura y financiamiento para la operación de esta red nacional de observatorios naturales.

Palabras clave: estudios socio-ecológico, cambio global, bienestar, toma de decisión.

\section{INTRODUCCIÓN}

Las sociedades humanas y los demás seres vivos que comparten el planeta dependen del funcionamiento y estado de la biosfera dentro de ciertos parámetros de estabilidad (Andersen et al. 2009, Chapin 2009, Folke et al. 2011). Diversas sociedades humanas han cohabitado y cohabitan en sus territorios de manera sustentable (Rozzi 2013). Sin embargo, la sociedad globalizada es hoy el principal agente transformador del paisaje y los ecosistemas (Wilkinson 2005, Ellis et al. 2013, Sandely Svenning 2013) y la velocidad a la que están ocurriendo estas transformaciones no tiene precedentes en la historia reciente del planeta Tierra (Wilkinson 2005, Steffen et al. 2011, Brando et al. 2014). Las prácticas de manejo para satisfacer las demandas de alimento y energía de una creciente población han desencadenado un proceso acelerado de cambio global que deriva en pérdidas de biodiversidad (Cardinale et al. 2012) y degradación de las funciones y servicios de los ecosistemas (Vitousek et al. 1997, MEA 2005), variables que actúan de manera sinérgica (Hooper et al. 2012). El deterioro paulatino y generalizado de las funciones ecosistémicas en el planeta disminuye su capacidad de proveer bienes y servicios a las sociedades actuales y futuras, afectando el bienestar humano (MEA 2005, Collins et al. 2011). Por tanto, existe una necesidad de idear formas de manejar los ecosistemas del planeta de manera sustentable en el largo plazo (Parris y Kates 2003, Chapin 2009).

Una red nacional de sitios de estudios socio-ecológicos de largo plazo contribuye a detectar y documentar las 
principales causas físicas y humanas que afectan el funcionamiento de los ecosistemas a nivel local, reconocer las tendencias de cambio a escala local, regional y global, y abordar el estudio de procesos y ciclos naturales que transcurren a escalas de tiempo superiores a una década (Armesto 1990, Robertson et al. 2012). Los resultados del monitoreo y estudio experimental de los ecosistemas a escalas decadales debe contribuir a descubrir tendencias de cambio imperceptibles en estudios de corto plazo, a diseñar y evaluar planes de manejo de los ecosistemas y a fundamentar la toma de decisiones en torno a la sustentabilidad (Likens y Lindenmayer 2011, Robertson et al. 2012, Carpenter et al. 2012).

El presente trabajo entrega una propuesta para el fortalecimiento y ampliación de la naciente Red Chilena de Sitios de Estudio Socio-ecológicos de Largo Plazo, LTSERChile, que ha sido impulsada, en una primera etapa, por el Instituto de Ecología y Biodiversidad (Anderson et al. 2010, Rozzi et al. 2012). Se propone un marco conceptual para la investigación de largo plazo en los ecosistemas nacionales, una propuesta de organización administrativa, y se discuten los requerimientos de infraestructura y financiamiento para la operación de la Red LTSER-Chile. También se consideran las necesidades de formación científica para aprovechar estos sitios de observación ecológica de largo plazo y para comunicar los resultados a la sociedad.

\section{MARCO CONCEPTUAL, PREGUNTAS Y OBJETIVOS DE LA RED}

Chile es un país que basa su economía principalmente en el uso de los recursos naturales. La falta de un manejo con un enfoque ecosistémico y de sustentabilidad generan el detrimento en las funciones ecosistémicas básicas tales como la regulación de caudales hidrológicos, purificación de agua, protección de suelos, la provisión de hábitats para la flora y la fauna, y la mantención de la productividad en el largo plazo (Vitousek et al. 1997, De Fries et al. 2004, MEA 2005). En este contexto, una red de estudios socioecológicos de largo plazo y de monitoreo de ecosistemas puede proveer líneas de base para evaluar el estado de deterioro o recuperación de los ecosistemas relevantes para la provisión de bienes y servicios a la sociedad, investigar experimentalmente los impactos de las prácticas de manejo, y apoyar al Estado en la formulación de estrategias para la adaptación y mitigación de los impactos del cambio global (Likens y Lindenmayer 2011, Sergeant et al. 2012). Como consecuencia de estos impactos, el Estado de Chile invierte hoy e invertirá en el futuro una gran cantidad de recursos económicos y humanos, los que pueden ahorrarse, en gran medida, a través del apoyo institucional a una red nacional de estudios socio-ecológicos con objetivos de largo plazo que aporte al conocimiento de las líneas de base y a resolver los problemas complejos relacionados a la integridad de los ecosistemas de los cuales los humanos son parte.
La inversión del Estado en una Red Nacional de sitios de investigación a largo plazo puede mejorarla gestión ambiental y los retornos económicos de la industria basada en los recursos naturales para el país, a través de la prevención y la detección temprana de tendencias climáticas y bióticas que afectan negativamente la producción agrícola, forestal y acuícola, finalmente, mediante la reducción de los costos ambientales provocados por las malas prácticas de manejo (ver Haughland et al. 2010, ejemplo en Canadá; Likens y Lindenmayer 2011, en Australia,). Esta Red Nacional, basada en sitios de estudio que abarquen una fracción relevante de la diversidad de ecosistemas del país, está orientada a obtener y comunicar evidencia científica crítica para la toma de decisiones, promover la gestión para conservación de la biodiversidad, monitorear los cambios en los ecosistemas que pueden afectar las funciones que sustentan la biodiversidad, la actividad económica y el bienestar humano.

En el contexto actual del cambio global acelerado, inducido por la actividad humana (Likens 1991, Sala et al. 2000, Ellis et al. 2013), y sus principales forzantes incluyendo cambios de uso del suelo, emisión de contaminantes a la atmósfera y las aguas, expansión de especies exóticas en hábitats nativos, homogenización a gran escala del paisaje y pérdidas de biodiversidad (Vitousek et al. 1997, MEA 2005), que también afectan los ecosistemas de Chile (CIREN 2010, Armesto et al. 2010, CONAF 2011, Universidad de Chile 2013), se propone las siguientes preguntas generales para fundamentar la investigación de la Red LTSER (figura 1): 1. ¿Cómo afectan los cambios de uso de suelo y la fragmentación de los hábitat nativos a los componentes, la estructura y el funcionamiento de la biodiversidad? 2. ¿Cuáles son las consecuencias de la variabilidad climática (intra e interanual) y las tendencias climáticas de largo plazo para la biodiversidad de los sistemas naturales y de aquellos manejados productivamente o manipulados experimentalmente, en los niveles de genes, especies y ecosistemas? 3. ¿Cómo las dinámicas de los ecosistemas alterados por el cambio global afectan la provisión de bienes y servicios para los seres humanos? 4. ¿Qué acciones humanas afectan los regímenes de perturbación natural de los ecosistemas, en términos de cambios en magnitud, frecuencia y tipos de perturbación? 5. ¿Cómo se puede amortiguar los impactos del cambio global y restaurar las funciones y procesos de los ecosistemas degradados? 6 . ¿Cómo se ven afectados los sistemas de vida y las sociedades humanas producto de la modificación antropogénica de las funciones ecosistémicas?

Estas preguntas generales pueden desglosarse en preguntas específicas que abordan problemas ambientales locales o regionales y que contribuyan a proponer soluciones de manejo, prevención, mitigación y reparación de daños ambientales. En este contexto, los objetivos específicos que se proponen para la red LTSER-Chile son: i) asegurar el monitoreo de variables críticas (indicadores) en toda la diversidad de ecosistemas (naturales y antropo- 


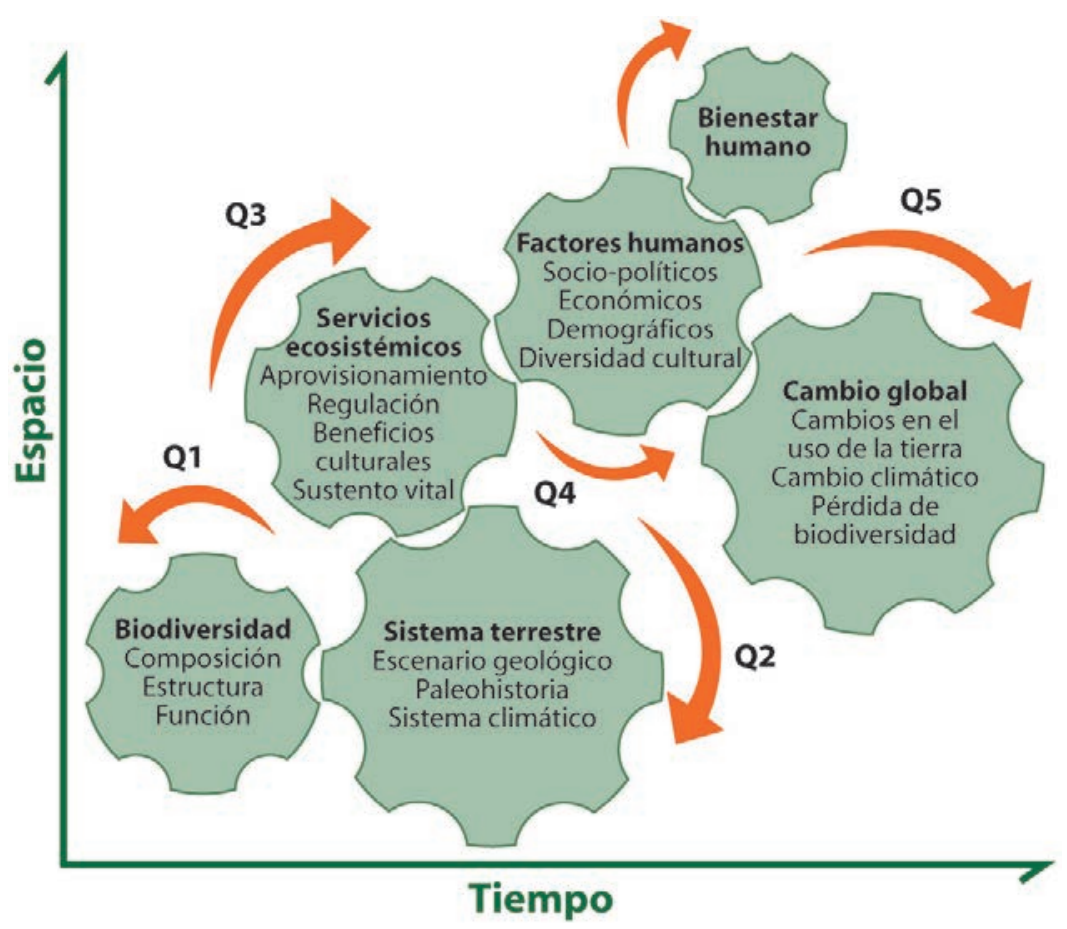

Figura 1. Un marco conceptual y preguntas de investigación propuestas para la Red Chilena de Estudios Socio-ecológicos de Largo Plazo (LTSER-Chile).

A conceptual framework and research questions proposed for the LTSER-Chile Network.

génicos) terrestres y marinos presentes en el país; ii) implementar y mantener infraestructura y equipamiento para conducir monitoreo y experimentación de largo plazo; iii) proveer un registro continuo y de calidad de variables sociales, biológicas y físicas relevantes como indicadores de tendencias ambientales y definir umbrales de cambio o colapso de los ecosistemas (Ostrom 2009, Collins et al. 2011, Palmer y Febria 2012); iv) desarrollar y mantener una plataforma eco-informática para el almacenamiento, procesamiento y acceso expedito a bases de datos de largo plazo, facilitando comparaciones con otros ecosistemas a escala local, regional y global (Luo et al. 2011); v) promover la integración disciplinaria de investigadores e instituciones científicas nacionales e internacionales; vi) ofrecer entrenamiento y capacitación a una nueva generación de estudiantes de pre y postgrado capaces de abordar la complejidad de los problemas socio-ecológicos, vii) generar espacios de diálogo donde los resultados de las investigaciones se presenten y discutan con la sociedad, estimulando nuevas preguntas de investigación pertinentes a la realidad local, y; vii) Integrar el trabajo a escala local con la red internacional de sitios de estudios ecológicos de largo plazo (ILTER-International Long Term EcologicalResearch Network), y otras redes mundiales de estudios de ecosistemas (e.g., Fluxnet, GLEON) que permita comparar e identificar tendencias globales.

De esta forma se espera contribuir a conectar y transmitir el conocimiento sobre un territorio hasta hoy ausente en el monitoreo de las tendencias climáticas y de impacto humano a escala global, cuales son los ecosistemas del cono sur de América (Rozzi et al. 2012).

\section{ORGANIZACIÓN DE LA RED LTSER-Chile}

Se propone que la red LTSER-Chile debe coordinar los esfuerzos de investigación en los sitios de estudios socioecológicos de largo plazo del país, atendiendo en forma colectiva los problemas conceptuales y logísticos. Es aconsejable que cada sitio esté dirigido por un científico de trayectoria reconocida, que coordine el trabajo que investigadores y estudiantes realizan en cada sitio (Likens y Lindenmayer 2011), promoviendo la conexión entre ciencia y sociedad a escala local y regional. Cada sitio de la Red debe tener su propio presupuesto, propia dinámica y autonomía, además de nexos institucionales, sin perder su vinculación con la red nacional a través de estudios comparativos y programas de intercambio de estudiantes e investigadores.

La red LTSER-Chile debe tener una figura legal y un estatuto para un funcionamiento ordenado. En cuanto a su organización (figura 2), cada sitio socio de la red LTSERChile podrá tener un representante para conformar la base societaria de la red, entre los cuales se elegirá un directorio. El Presidente y otros cargos del directorio deben ser elegidos cada dos o tres años. La tarea del directorio sería representar a los sitios a escala nacional y vincular la red LTSER-Chile con la red I-LTER y otras redes a escala con- 


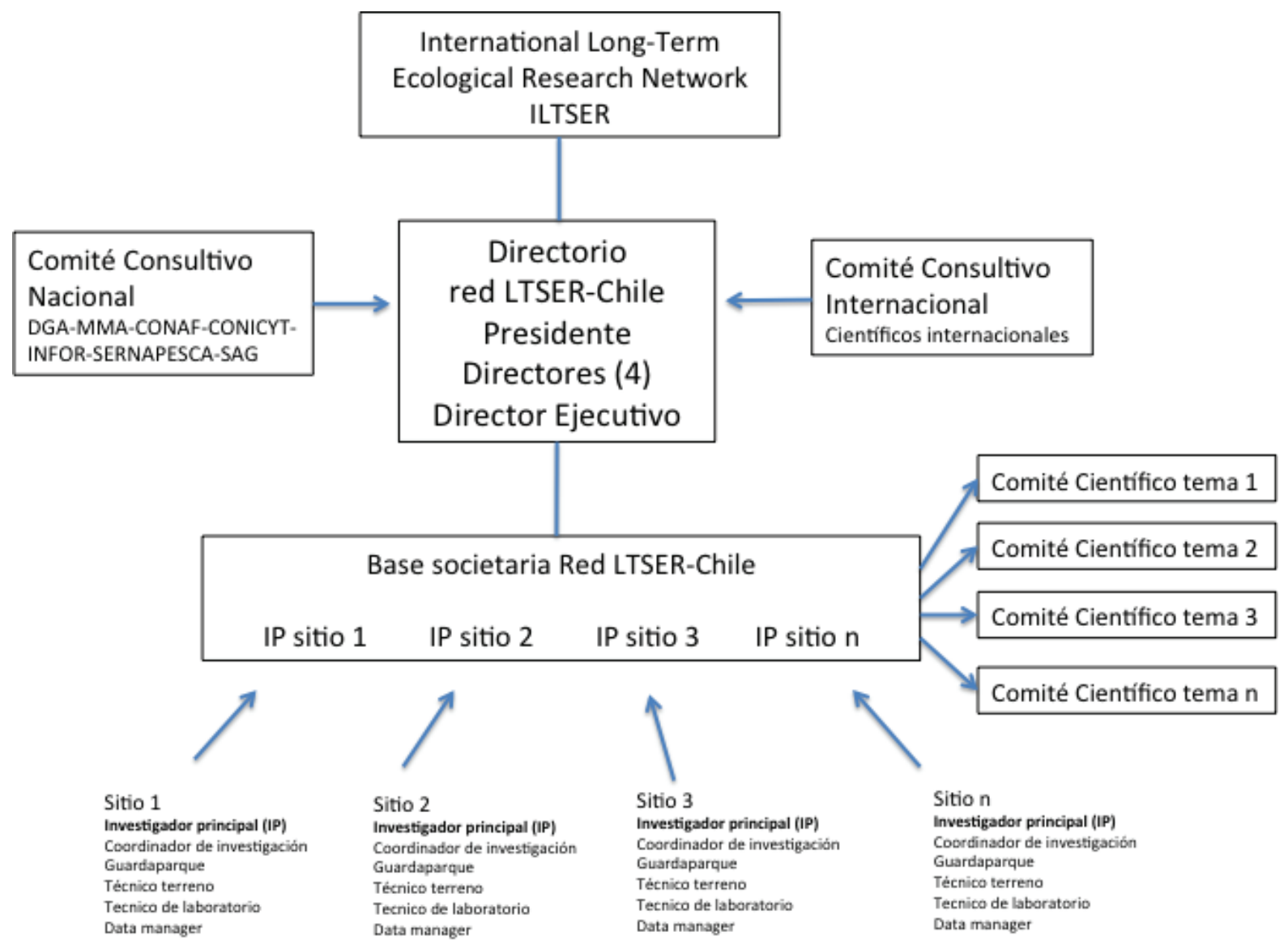

Figura 2. Organigrama de la Red Chilena de Estudios Socio-Ecológicos de Largo Plazo.

Organizational framework for the LTSER-Chile Network.

tinental o global. El directorio debe asegurar, mediante organización de talleres, cursos, conferencias y otros medios, que los resultados de las investigaciones de la Red puedan ser conocidos por los tomadores de decisiones y encargados de planificación y manejo de los recursos naturales a escala local y nacional. El equipo de apoyo al directorio debería incluir un director ejecutivo, un encargado de la plataforma eco-informática y personal técnico especialista en investigación y monitoreo de ecosistemas. El director ejecutivo deberá llevar a cabo los lineamientos trazados por el directorio, generando proyectos colaborativos para lograr los objetivos de la red LTSER y buscar financiamiento público y privado, nacional e internacional, con el objeto de asegurar la sostenibilidad financiera e institucional en el largo plazo. La plataforma eco-informática es la base para mantener los datos de largo plazo, por ende, el encargado de esta plataforma es de vital importancia para asegurar la permanencia de la información.

Los representantes de cada sitio pueden crear comisiones permanentes o transitorias para abordar temas específicos vinculados a las preguntas de investigación, el marco conceptual de la red LTSER-Chile y las necesidades urgentes del país.
Una vez establecida la red, sería recomendable formar dos comités consultivos que trabajen en asociación con el directorio, uno de carácter nacional y otro internacional. En el comité nacional deben estar representados diversos servicios y organismos públicos relacionados con la gestión ambiental del Estado (MMA, CONAF, DGA, SAG, SERNAPESCA), quienes pueden contribuir a orientar las investigaciones científicas de la red LTSER de acuerdo a las necesidades de cada sector y promover la vinculación de los resultados de estas investigaciones con las políticas públicas. El comité internacional estaría constituido por científicos internacionales con experiencia en investigación socio-ecológica de largo plazo y vinculados a otras redes globales, cuyo rol sería colaborar en incrementar los estándares de calidad de la investigación científica de la red LTSER-Chile, actualizar al directorio sobre las tendencias globales y posicionar nuestros resultados en el ámbito de la investigación a escala global.

\section{NECESIDADES DE FORMACIÓN}

Para fortalecer la red de estudios socio-ecológicos a largo plazo es indispensable formar a los nuevos científicos 
y profesionales con una visión integral y holista, que reconozcan y valoren la importancia de la integración de las diversas disciplinas, principalmente del área ecológica y social, para analizar y proponer soluciones a problemáticas socio-ecológicas complejas. Sin embargo, hoy este enfoque no se aplica en la formación tradicional de la mayoría de las universidades chilenas. Para abordar problemas complejos es preciso reforzar las habilidades necesarias para el trabajo en equipos, el pensamiento integrado y la síntesis (Morin 1990). El pensamiento complejo se nutre de todas las ciencias, especialmente de las ciencias biológicas, físicas, matemáticas, y las ciencias humanas, las artes y las humanidades. Ahora bien, la integración de tales ciencias no es simple, ya que el pensamiento complejo no es una réplica de las formas habituales de organización del conocimiento y métodos de investigación. Al contrario, el pensamiento complejo toma distancia de los métodos convencionales que habitualmente separan y distinguen las disciplinas (Morin 1990). En este contexto, para favorecer la formación transdisciplinaria a nivel de pre y postgrado es relevante crear programas de estudio entre facultades de distintas universidades y centros de investigación, que permitan abordar problemas socialmente relevantes y de interés común, generando nuevos enfoques metodológicos y conceptuales, integrando la teoría y práctica. La red LTSER-Chile debe aportar con los espacios y generar oportunidades de interacción entre estudiantes, investigadores y profesionales de distintas disciplinas, con los habitantes de los territorios donde se realiza investigación socio-ecológica, que permita acercar la teoría a la práctica.

\section{CIENCIA Y SOCIEDAD}

Dado que el desarrollo económico y social depende, en gran medida, de sistemas productivos basados en bienes y servicios derivados de los ecosistemas (De Fries et al. 2004, Tallis et al. 2008), un objetivo central de la red LTSERChile debe ser impulsar esquemas de desarrollo basados en sistemas productivos locales sustentables. Por ende, el éxito de la red LTSER-Chile depende en gran medida de su capacidad de vincularse con los diversos sectores sociales que habitan y mantienen actividades productivas en los diversos territorios del país. En este contexto, los sistemas de vida locales son el nexo más directo entre la población humana y los ecosistemas (Tyler 2006), ya que son dinámicos, evolucionan y están abiertos a la innovación, se adaptan constantemente a los cambios ambientales y demográficos, por lo que pueden constituir el foco de la investigación socioecológica de largo plazo (Carpenter et al. 2012). El monitoreo de largo plazo de los sistemas socio-ecológicos facilita la evaluación de los cambios generados por las comunidades locales y el análisis de las consecuencias de diferentes prácticas de manejo (Keough y Blahna 2006, Lindenmayer y Likens 2009). Para hacer operativa la interacción entre los sitios de estudio de la red LTSER y los habitantes locales, con el propósito de cambiar las formas de manejo y uso del entorno, se requiere diseñar nuevos mecanismos o utilizar herramientas de planificación existentes (Planes de Desarrollo Comunal), que deben incluir metas de sustentabilidad acordes a los principios del manejo adaptativo (Stankey et al. 2005, Keith et al. 2011), fundamentado en experimentos y el monitoreo de largo plazo. Finalmente, las organizaciones locales y los centros académicos pueden colaborar para fortalecer los programas de investigación y aumentar su incidencia en la toma de decisiones.

\section{INFRAESTRUCTURA Y FINANCIAMIENTO}

La mantención de la investigación socio-ecológica en el tiempo requiere de un respaldo financiero e institucional sólido que pueda dar continuidad a cada sitio. Los costos asociados a la implementación y operación de una red de sitios de estudio socio-ecológico de largo plazo son altos (cuadro 1), pero corresponden a una inversión con retornos significativos en términos de conocimiento y desarrollo de procesos productivos sustentables y la prevención y mitigación de impactos ambientales.

Se ha estimado que el costo de instalación de un sitio de estudio socio-ecológicos de largo plazo, que cuente con

Cuadro 1. Presupuesto para la inversión inicial de infraestructura para un sitio de estudio socio-ecológico de largo plazo y los costos de operación anual para la gestión de la red LTSER.

Minimum budget required for installing a long-term socioecological study site and network annual management requirement.

\begin{tabular}{|c|c|}
\hline Infraestructura (inversión inicial) & Costo unitario $\left(\mathrm{M} \$^{*}\right)$ \\
\hline $\begin{array}{l}\text { Edificación (residencia, salón de uso } \\
\text { múltiple y laboratorio) }\end{array}$ & 87.000 \\
\hline Estación meteorológica & 8.000 \\
\hline Estación limnimétrica & 8.000 \\
\hline $\begin{array}{l}\text { Parcelas permanentes y } \\
\text { experimentación }\end{array}$ & 40.000 \\
\hline Vehículo todo terreno & 15.000 \\
\hline $\begin{array}{l}\text { Instrumental y equipos de } \\
\text { investigación }\end{array}$ & 120.000 \\
\hline $\begin{array}{l}\text { Instrumental y equipos de } \\
\text { laboratorio }\end{array}$ & 200.000 \\
\hline Total Infraestructura (M\$) & 478.000 \\
\hline $\begin{array}{l}\text { Gestión Red LTSER } \\
\text { (operación anual) }\end{array}$ & Costo unitario (M\$) \\
\hline $\begin{array}{l}\text { Personal (director ejecutivo, } \\
\text { administrador y técnicos) }\end{array}$ & 52.000 \\
\hline Transporte y viáticos & 10.000 \\
\hline $\begin{array}{l}\text { Mantenciones y Fungibles } \\
\text { (insumos, materiales y repuestos) }\end{array}$ & 11.000 \\
\hline Total Operaciones (M\$ año-1) & 73.000 \\
\hline
\end{tabular}

* M\$ = miles de pesos chilenos. 
facilidades logísticas básicas para recibir investigadores, estudiantes y comunidades locales, junto con el desarrollo de investigación y monitoreo, asciende a la suma de 478 millones de pesos (cuadro 1). Para cada sitio este costo varía de acuerdo a las condiciones de aislamiento y rigurosidad ambiental en que se emplaza. En general, es recomendable buscar la instalación de las estaciones en sitios accesibles que cuenten con suministro eléctrico para facilitar su operación.

Los costos que se requieren para gestionar la red LTSER- Chile en su conjunto ascienden a los 73 millones de pesos anuales, de los cuales 52 millones corresponden a contratación de personal (dirección ejecutiva, plataforma eco-informática y administración) y 21 millones a operación (viajes de coordinación, organización de reuniones de trabajo nacionales e internacionales y mantención de la plataforma eco-infomática). La plataforma eco-informática requiere una inversión inicial cercana a los cinco millones de pesos, y un costo de mantención y actualización aproximado de dos millones de pesos anuales.

\section{EXPECTATIVAS FUTURAS}

Sala et al. (2000) analizaron diversos escenarios futuros y sus efectos en la biodiversidad en los diferentes biomas, concluyendo que los ecosistemas mediterráneos y templados, están entre los más amenazados del mundo debido al cambio de uso de suelo generados por las sociedades humanas. Considerando la amplia representación de estos ecosistemas en Chile, es urgente contar con un monitoreo y experimentos de largo plazo que permitan evaluar futuros escenarios socio-económicos y su impacto sobre los componentes de la biodiversidad.

La sociedad del siglo XXI exige importantes desafíos de gestión ambiental para asegurar la sustentabilidad, que consideren simultáneamente la demanda socio-económica de bienes y servicios con la mantención de la biodiversidad que sustenta la productividad de los ecosistemas (De Fries et al. 2004). La meta es entender los múltiples factores que influ- yen en la mantención de los bienes y servicios de cada ecosistema (interacción entre los procesos suelo-planta y los ciclos biogeoquímicos), lo cual solo puede abordarse en estudios a largo plazo y modelos de sistemas complejos (Holling 2001).

El estudio a largo plazo de las prácticas de manejo y el manejo adaptativo deben estar enfocados en procesos ecosistémicos relevantes, como por ejemplo, la regulación de los caudales hidrológicos y los ciclos biogeoquímicos (Likens 1991). El manejo adaptativo de ecosistemas involucra un aprendizaje a través de experimentos rigurosos que permiten mejorar las prácticas productivas (Holling 2001) y vincular estas prácticas con las políticas públicas (Stankey et al. 2005). En muchos sentidos, este último componente es donde el concepto adaptativo opera, es decir, es esencial la retroalimentación y aplicación de los resultados de la experimentación a la práctica.

Esta propuesta sitúa a los usuarios de los ecosistemas en el centro mismo de los proyectos de investigación, como participantes tanto en la práctica como en el aprendizaje (Tyler 2006). Las estrategias de investigación deben contribuir a mejorar los sistemas económicos locales sobre la base de la participación de los usuarios de los ecosistemas. En este contexto, el conocimiento tradicional que poseen las comunidades sobre los ecosistemas donde han habitado por siglos o milenios debe complementar al conocimiento científico. Los valores y creencias son parte del sistema de conocimientos que integra la ciencia con una ética práctica (Berkes et al. 1998).

La Red LTSER-Chile en el futuro debería incluir una representación de los diversos ecosistemas del país, fortalecer el análisis, la síntesis y la capacidad predictiva a través de la integración de científicos, comunidades locales, el sector público y privado. Debería aportar a la identificación de las estrategias óptimas para la gestión del territorio con el resguardo de la biodiversidad, junto con la planificación y uso sustentable de los recursos naturales. Un buen funcionamiento de esta red nacional contribuiría a generar un cambio hacia un nuevo paradigma social

Cuadro 2. Hacia un cambio de paradigma social.

Towards a social paradigm shift.

\begin{tabular}{|c|c|}
\hline Visión clásica & Nuevo paradigma \\
\hline $\begin{array}{l}\text { El ser humano se siente disgregado de la naturaleza y } \\
\text { por sobre ella }\end{array}$ & $\begin{array}{l}\text { El ser humano se reconoce como parte del ecosistema, en el tiempo } \\
\text { y en el espacio }\end{array}$ \\
\hline Visión de corto plazo y local & Visión de largo plazo, local y global \\
\hline La biodiversidad es sólo de interés privado o particular & $\begin{array}{l}\text { La biodiversidad es de interés público y considerada como estratégica } \\
\text { para la Seguridad Nacional }\end{array}$ \\
\hline $\begin{array}{l}\text { El poder está en un reducido número de seres humanos } \\
\text { que asumen que los recursos naturales son ilimitados } \\
\text { en el tiempo y el espacio }\end{array}$ & $\begin{array}{l}\text { La sociedad en su conjunto reconoce, valora y protege los bienes y } \\
\text { servicios de la biodiversidad para hoy y las futuras generaciones }\end{array}$ \\
\hline Las prácticas productivas degradan los ecosistemas & Las prácticas productivas aportan a la conservación de la biodiversidad \\
\hline $\begin{array}{l}\text { Solo una parte de la población accede a los bienes y } \\
\text { servicios básicos de calidad }\end{array}$ & $\begin{array}{l}\text { En la sociedad existe equidad y acceso a los bienes y servicios } \\
\text { básicos de calidad }\end{array}$ \\
\hline
\end{tabular}


(cuadro 2), donde el ser humano se reconoce como parte del ecosistema, se planifica con una visión de largo plazo y a múltiples escalas; donde la biodiversidad es considerada un patrimonio de interés público; donde las prácticas productivas adaptativas se basan en el conocimiento científico y el conocimiento ancestral para contribuir a la sustentabilidad; donde la sociedad conoce, valora y protege la biodiversidad, en una sociedad más justa, con equidad y acceso a los bienes y servicios básicos de calidad.

\section{AGRADECIMIENTOS}

Agradecemos a Sebastián Abades por su ayuda en estimar los costos mínimos de una plataforma eco-informática. El trabajo de la Red LTSER del Instituto de Ecología y Biodiversidad ha sido financiado por ICM (Proyecto P05-002) y CONICYT (Proyecto Financiamiento Basal PFB-23). Este trabajo es una contribución al programa de investigación de la Red LTSER-Chile.

\section{REFERENCIAS}

Andersen T, J Carstensen, E Hernández-García, CM Duarte. 2009. Ecological thresholds and regime shifts: approaches to identification. Trends in Ecology \& Evolution 24: 49-57.

Anderson CP, R Rozzi, JJ Armesto, JR Gutiérrez.2010. Construyendo una Red Chilena para Estudios Socioecológicos a Largo Plazo: Avances, enfoques y relevancia. Revista Chilena de Historia Natural 83: 1-11.

Armesto JJ. 1990. Estudio a largo plazo: Una prioridad para la investigación ecológica de hoy. Revista Chilena de Historia Natural 63: 7-9.

Armesto JJ, D Manuschevich, A Mora, C Smith-Ramirez, R Rozzi, AM Abarzúa, PA Marquet. 2010. From the Holocene to the Anthropocene: A historical framework for land cover change in southwestern South America in the past 15,000 years. Land Use Policy 27: 148-160.

Berkes F, M Kislalioglu, C Folke y M Gadgil. 1998. Exploring the basic ecological unit: ecosystem-like concepts in traditional societies. Ecosystems 1: 409-415.

Brando PM, JK Balch, DC Nepstad, DC Morton, FE Putz, MT Coe, D Silvério, MN Macedo, EA Davidson, CC Nóbrega, A Alencara, BS Soares-Filho. 2014. Abrupt increases in Amazonian tree mortality due to drought-fire interactions. Proceedings of the National Academy of Sciences of the United States of America 111: 6347-52.

Cardinale BJ, JE Duffy, A Gonzalez, DU Hooper, C Perrings, P Venail, A Narwani, GM Mace, D Tilman, DA Wardle, AP Kinzig, GC Daily, M Loreau, JB Grace, A Larigauderie, DS Srivastava, S Naeem. 2012. Biodiversity loss and its impact on humanity. Nature 486: 59-67.

Carpenter S, C Folke, A Norstrom, O Olsson, L Schultz, B Agarwal, P Balvanera, B Campbell, JC Castilla, W Cramer, R De Fries, P Eyzaguirre, TP Hughes, S Polasky, Z Sanusi, R Scholes, M Spierenburg. 2012. Program on ecosystem change and society: an international research strategy for integrated social-ecological systems. Current Opinion in Environmental Sustainability 4(1): 134-138.

Chapin FS. 2009. Managing Ecosystems Sustainably: The Key
Role of Resilience. In Chapin FS, GP Kofinas, C Folke eds. Principles of Ecosystem Stewardship. Resilience-based Natural Resource Management in a Changing World. New York, USA. Springer. p. 29-53.

CIREN (Centro de Información de Recursos Naturales, CL). 2010. Determinación de Erosión Actual y Potencial para el Territorio de Chile. Síntesis de Resultados. Santiago, Chile. CIREN. 60 p.

Collins SL, SR Carpenter, SM Swinton, DE Orenstein, DL Childers, TL Gragson, NB Grimm, JM Grove, SL Harlan, JP Kaye, AK Knapp, GP Kofinas, JJ Magnuson, WH McDowell, JM Melack, LA Ogden, GP Robertson, MD Smith, AC Whitmer. 2011. An integrated conceptual framework for long-term social-ecological research. Frontiers in Ecology and the Environment 9(6): 351-357.

CONAF (Corporación Nacional Forestal, CL). 2011. Catastro de los Recursos Vegetacionales Nativos de Chile. Monitoreo de Cambios y Actualizaciones. Período 1997 - 2011. Santiago, Chile. CONAF. 25 p.

De Fries R, J Foley, G Asner. 2004. Land-Use Choices: Balancing Human Needs and Ecosystem Function. Frontiers in Ecology and the Environment 2(5): 249-257.

Ellis EC, JO Kaplan, DQ Fuller, S Vavrus, KK Goldewijk, PH Verburg. 2013. Used planet: A global history. Proceedings of the National Academy of Sciences of the United States of America 110: 7978-85.

Folke C, A Jansson, J Rockström, P Olsson, SR Carpenter, FS Chapin, AS Crépin , G Daily, K Danell, J Ebbesson, T Elmqvist, V Galaz, F Moberg, M Nilsson, H Osterblom, E Ostrom, A Persson, G Peterson, S Polasky, W Steffen, B Walker, F Westley. 2011. Reconnecting to the biosphere. Ambio 40: 719-738.

Haughland DL, JM Hero, J Schieck, JG Castley, S Boutin, P Sólymos, BE Lawson, G Holloway, WE Magnusson. 2010. Planning forwards: biodiversity research and monitoring systems for better management. Trends in Ecology \&Evolution 25: 199-200.

Holling C. 2001. Understanding the Complexity of Economic, Ecological, and Social Systems. Ecosystems 4: 390-405

Hooper DU, EC Adair, BJ Cardinale, JE Byrnes, BA Hungate, KL Matulich, A Gonzalez, JE Duffy, L Gamfeldt, MI O’Connor. 2012. A global synthesis reveals biodiversity loss as a major driver of ecosystem change. Nature 486: 105-108.

Keith DA, TG Martin, E McDonald-Madden, C Walters. 2011. Uncertainty andadaptive management for biodiversity conservation. Biological Conservation 144: 1175-1178.

Keough HL, DJ Blahna. 2006. Achieving integrative, collaborative ecosystem management. Conservation Biology 20: 1373-82.

Likens G, D Lindenmayer. 2011. A strategic plan for an Australian Long-Term Environmental Monitoring Network. Austral Ecology 36: 1-8.

Likens G. 1991. Human-Accelerated Environmental Change. BioScience 41(3): 130.

Lindenmayer DB, GE Likens. 2009. Adaptive monitoring: a new paradigm for long-term research and monitoring. Trends in Ecology \& Evolution 24(9): 482-486.

Luo Y, J Melillo, S Niu, C Beier, J Clark, A Classen, E Davidson, JS Dukes, RD Evans, CB Field, CI Czimczik, M Keller, BA Kimball, LM Kueppers, RJ Norby, SL Pelini, E Pendall, E 
Rastetter, J Six, M Smith, MG Tjoelker, MS Torn. 2011. Coordinated approaches to quantify long-term ecosystem dynamics in response to global change. Global Change Biology 17: 843-854.

MEA (Millennium Ecosystem Assessment, US). 2005. Ecosystems and Human Well-being: Synthesis. Washington D.C., USA. Island Press. 102 p.

Morin E. 1990. Introducción al pensamiento complejo. Barcelona, España. Editorial Gedisa. 164 p.

Ostrom E. 2009. A General Framework for Analyzing Sustainability of Social-Ecological Systems. Science 325: 419-422.

Palmer MA, CM Febria. 2012. Ecology. The heartbeat of ecosystems. Science 336: 1393-1394.

Parris T, R Kates. 2003. Characterizing a sustainability transition: Goals, targets, trends, and driving forces. Proceedings of the National Academy of Sciences 100: 8068-8073.

Robertson GP, SL Collins, DR Foster, N Brokaw, HW Ducklow, TL Gragson, C Gries, SK Hamilton, AD McGuire, JC Moore, EH Stanley, RB Waide, MW Williams. 2012. Long-Term Ecological Research in a Human-Dominated World. BioScience 62(4): 342-353

Rozzi R. 2013. Biocultural Ethics: From Biocultural Homogenization toward Biocultural Conservation. In Rozzi R, STA Pickett, C Palmer, JJ Armesto, JB Callicott eds. Linking Ecology and Ethics for a Changing World: Values, Philosophy, and Action. New York, USA. Springer. p. 9-32.

Rozzi R, JJ Armesto, JR Gutiérrez, F Massardo, GE Likens, CB Anderson, A Poole, KP Moses, Eugene Hargrove, AO Mansilla, JH Kennedy, M Willson, K Jax, CG Jones, JB Callicott, MTK Arroyo. 2012. Integrating ecology and environmental ethics: earth stewardship in the southern end of the Americas. BioScience 62(3): 226-236.

Sala OE, FS Chapin III, JJ Armesto, R Berlow, J Bloomfield, R Dirzo, E Huber-Sanwald, LF Huenneke, RB Jackson, A
Kinzig, R Leemans, D Lodge, HA Mooney, M Oesterheld, NL Poff, MT Sykes, BH Walker, M Walker, DH Wall. 2000. Global biodiversity scenarios for the year 2100. Science 287: 1770-1774.

Sandel B, JCC Svenning. 2013. Human impacts drive a global topographic signature in tree cover. Nature Communications 4: 2474.

Sergeant C, BJ Moynahan, WF Johnson. 2012. Practical advice for implementing long-term ecosystem monitoring. Journal of Applied Ecology 49 (5): 969-973.

Stankey G, R Clark, B Bormann. 2005. Adaptive management of natural resources: theory, concepts, and management institutions. General Technical Report PNW-GTR-654. Portland, OR: U.S. Department of Agriculture, Forest Service, Pacific Northwest Research Station. 80 p.

Steffen W, J Grinevald, P Crutzen, J McNeill. 2011. The Anthropocene: conceptual and historical perspectives. Philosophical transactions of the Royal Society. Series A, Mathematical, Physical, And Engineering Sciences 369: 842-67.

Tallis H, P Kareiva, M Marvier, A Chang. 2008. An ecosystem services framework to support both practical conservation and economic development. Proceedings of the National Academy of Sciences of the United States of America 105: 9457-64.

Tyler S. 2006. Co-management of natural resources: local learning for poverty reduction. Ottawa, Ontario, Canadá. Centro Internacional de Investigaciones para el Desarrollo. 80 p.

Universidad de Chile. 2013. Informe País: Estado del medioambiente en Chile 2012. Santiago, Chile. Centro de Análisis de Políticas Públicas del Instituto de Asuntos Públicos. 296 p.

Vitousek P, H Mooney, J Lubchenco, J Melillo. 1997. Human domination of earth's ecosystems. Science 277: 494-499.

Wilkinson B. 2005. Humans as Geologic Agents: A deep-time perspective. Geology 33(3): 161-164.

Recibido: 10.07.14

Aceptado: 14.10 .14 